INPLASY

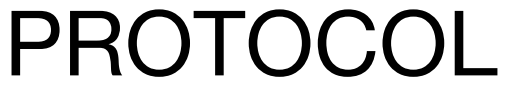

To cite: Wang et al. Efficacy of CoQ10 supplementation for the treatment of primary CoQ10 deficiency. Inplasy protocol 202220113. doi: 10.37766/inplasy2022.2.0113

Received: 25 February 2022

Published: 25 February 2022

Corresponding author: Siegfried Hekimi

siegfried.hekimi@mcgill.ca

Author Affiliation:

McGill University.

Support: CIHR.

Review Stage at time of this submission: Data extraction.

Conflicts of interest:

None declared.

\section{Efficacy of CoQ10 supplementation for the treatment of primary CoQ10 deficiency}

\author{
Wang, Y1; Hekimi, S².
}

Review question / Objective: How effective is oral CoQ10 supplementation to treat primary CoQ10 deficiency?

Condition being studied: Coenzyme Q10 (CoQ10), also known as ubiquinone, is an essential component of the mitochondrial respiratory chain. CoQ10 is also known to be involved in several other cellular functions besides the respiratory chain. All cells rely exclusively on endogenous CoQ synthesis. So far, $13 \mathrm{COQ}$ genes whose products participate in CoQ10 biosynthesis have been identified in humans. Mutations in COQ genes cause primary CoQ10 deficiency (PUD), a rare, clinically heterogenous, disorder. Symptoms are those of inborn mitochondrial respiratory chain disorders, including early onset, multi-organ involvement, and prevalence of neurological and muscular manifestations. With the increasing availability and affordability of genomic sequencing technology, more and more PUD patients and novel PUD disease variants are being reported. CoQ10 supplementation as replacement therapy is frequently initiated immediately after diagnosis, and the majority of the literature on CoQ10 deficiency claims that this treatment is effective. However, there is lack of clear evidence for this claim. The planned review aims to identify, summarize and evaluate all the available evidence for the effectiveness of CoQ10 supplementation for the treatment of PUD.

INPLASY registration number: This protocol was registered with the International Platform of Registered Systematic Review and Meta-Analysis Protocols (INPLASY) on 25 February 2022 and was last updated on 25 February 2022 (registration number INPLASY202220113).

\section{INTRODUCTION}

Review question / Objective: How effective is oral CoQ10 supplementation to treat primary CoQ10 deficiency?
Rationale: Coenzyme Q10 (CoQ10), also known as ubiquinone (UQ10) is a mobile component of the mitochondrial respiratory 
chain. It is also known to play a role in several other essential cellular processes including ROS generation and detoxification. Mutations in CoQ10 biosynthetic genes cause primary CoQ10 deficiency (PUD), a rare inborn disease which predominantly manifests as a mitochondrial disorder. CoQ10 deficiency status has also been described in association with a variety of other disease conditions, referred to as secondary CoQ deficiency. The prevailing literature states that, CoQ10 deficiency is treatable by oral supplementation, yet there is a lack of clear supporting evidence for the view. No randomized, controlled trials have been conducted to date on the efficacy of CoQ10 supplementation to treat CoQ10 deficiency.

Condition being studied: XCoenzyme Q10 (CoQ10), also known as ubiquinone, is an essential component of the mitochondrial respiratory chain. CoQ10 is also known to be involved in several other cellular functions besides the respiratory chain. All cells rely exclusively on endogenous CoQ synthesis. So far, $13 \mathrm{COQ}$ genes whose products participate in CoQ10 biosynthesis have been identified in humans. Mutations in COQ genes cause primary CoQ10 deficiency (PUD), a rare, clinically heterogenous, disorder. Symptoms are those of inborn mitochondrial respiratory chain disorders, including early onset, multi-organ involvement, and prevalence of neurological and muscular manifestations. With the increasing availability and affordability of genomic sequencing technology, more and more PUD patients and novel PUD disease variants are being reported. CoQ10 supplementation as replacement therapy is frequently initiated immediately after diagnosis, and the majority of the literature on CoQ10 deficiency claims that this treatment is effective. However, there is lack of clear evidence for this claim. The planned review aims to identify, summarize and evaluate all the available evidence for the effectiveness of CoQ10 supplementation for the treatment of PUD.

\section{METHODS}

Search strategy: A literature search will be performed in PubMed for studies that described primary CoQ10 deficiency patients, up until March 2022. The references cited in the articles identified will be manually screened for any additional relevant study. We will impose no publication status or language restrictions and will consider all types of studies regardless of research design.

Participant or population: We will perform a literature search to identify studies that describe primary CoQ10 deficiency patients and extract information such as age of onset, major symptoms, age at the last reported exam or death, molecular lesions in $\mathrm{COQ}$ genes or proteins, severity of CoQ10 deficit, CoQ10 treatments received, and clinical outcomes. Our analysis will exclude studies where no data on patient treatment with CoQ10 is provided, or if patients were treated but outcome data is not reported, or the reported effects are contradictory or ambiguous. No other restrictions will be introduced including CoQ10 dosage (dosage, formulation, dosing frequency), time of initial treatment, duration of treatment, or concurrent treatments.

Intervention: Administration of any form of CoQ10 (including structural analogues of CoQ10) for any time of duration with any treatment regimen.

Comparator: Data on untreated control patients are not available. All reported effects are comparison of the clinical condition of patients before and after treatment.

Study designs to be included: All PUD patients that have been reported and have been treated with CoQ10 after diagnosis, and whose responses to the treatment were sufficiently described for an assessment of whether there were effects of the treatment.

Eligibility criteria: Our study will include all PUD patients that have been reported and 
have been treated with CoQ10 after diagnosis, and whose responses to the treatment were sufficiently described for an assessment of whether there were effects of the treatment. We will exclude all studies in which no data on patient treatment is provided, or when the outcome of treatment is not reported, or when the reported effects are contradictory or ambiguous.

Information sources: Searches will be performed at PubMed/Medline for studies that described PUD patients up to March 2022. In addition, references of identified studies will be manually screened to search additional relevant studies.

Main outcome(s): Any effect on patients' clinical status including effects on quantitative measures (biochemical tests, physiological measures, and behavioral assessments), as well as subjective descriptions of the patients' condition before and after CoQ10 treatment

Quality assessment / Risk of bias analysis: Two reviewers will independently assess the quality of the data on the outcome of CoQ10 treatment for each eligible study. If contradictory or discrepant findings are described in the original report (partial improvement of some symptoms but worsening of others) or when outcome descriptions are ambiguous (partial improvement of only one symptom and without relying on any quantifiable measure), we will consider the data of low quality or having a high risk of bias. The study will therefore be excluded. Any disagreement will be settled by discussion.

Strategy of data synthesis: We will differentiate the outcome of CoQ10 treatment for each eligible PUD patient into responding and non-responding. "Nonresponding" will include the cases in which no response to treatment is reported or when the responses lack convincing demonstration of an objective response to CoQ10. The responding group will include all cases in which quantitative or semiquantitative measures suggest improvement of the disease pathology as well as cases where clinical improvement of at least 2 symptoms are noted at a posttreatment follow up. Patient counts in each group will be presented.

Subgroup analysis: Not Applicable.

Sensitivity analysis: Not Applicable.

Language: English.

Country(ies) involved: Canada.

Keywords: Coenzyme $Q$, ubiquinone, CoQ10, CoQ10 biosynthesis, CoQ10 deficiency, CoQ10 supplementation, inborn mitochondrial disorders.

Contributions of each author:

Author 1 - Siegfried Hekimi.

Email: siegfried.hekimi@mcgill.ca

Author 2 - Ying Wang.

Email: ying.wang10@mcgill.ca 